\title{
DETECTION OF LANGMUIR SOLITONS: IMPLICATIONS FOR TYPE III BURST EMISSION MECHANISMS AT $2 \omega_{P E}$
}

\author{
G. THEJAPPA, * R. G. STONE and M. L. GOLDSTEIN \\ NASA, Goddard Space Flight Center, Greenbelt MD 20771
}

\begin{abstract}
We present the experimental verification of existing theoretical models of emission mechanisms of solar type III bursts at the second harmonic of the plasma frequency, $\omega_{\text {pe }}$. This study is based on the detection of Langmuir and envelope solitons by the Ulysses spacecraft inside three type III burst source regions. We show that the oscillating-twostream instability, coherent radiation by Langmuir solitons and stochastic phase mixing of the Langmuir waves in the strong turbulence regime are the appropriate emission mechanisms at $2 \omega_{p e}$.
\end{abstract}

Key words: Langmuir Solitons - Type III Bursts

\section{Introduction}

The identification of the mechanisms responsible for: (1) stabilizing the type III electron beams and (2) conversion of Langmuir waves into electromagnetic radiation at the fundamental of the plasma frequency, $\omega_{p e}$, and its second harmonic, $2 \omega_{p e}$, is one of the outstanding problems in solar radio astronomy. The Ulysses high time resolution observations of Langmuir waves associated with local type III bursts indicate that the strong turbulence processes, such as the modulational instability, the oscillating two-stream instability, Langmuir soliton formation and Langmuir collapse dominate not only the emission processes at $\omega_{p e}$, but also beam stabilization processes (Thejappa et al., 1993, 1995a and b). The purpose of the present paper is to identify the mechanisms responsible for the type III emission at $2 \omega_{p e}$. The observations and theoretical analysis will be presented in sections 2 and 3 , respectively, which will be summarized in Table 1.

\section{Observations}

The Unified Radio and Plasma Wave Experiment (Stone et. al., 1992) on the Ulysses spacecraft has detected three local type III bursts (Reiner et al., 1992, Thejappa et. al., 1993, 1995a, b and c). In Fig. 1a, we present the wave activity associated with one of these events. The top two panels

* Also at Astronomy Department, University of Maryland, College Park, MD 20742; On leave from Indian Institute of Astrophysics, Bangalore-560034, INDIA 
show the type III burst electric field at $\simeq 27.5$ and $\simeq 15.5 \mathrm{kHz}$ respectively. The third panel shows the Langmuir waves at $13.8 \mathrm{kHz}$ with a $16 \mathrm{~s}$ resolution. The fourth panel shows the ion-acoustic-like wave activity at $\mathbf{2 . 3}$ $\mathrm{kHz}$. The fifth and sixth panels show the peak electric field data at 112 and $2.7 \mathrm{~Hz}$, respectively. Fig. $1 \mathrm{~b}$ presents the typical high time resolution $(\simeq 1$ $\mathrm{ms}$ ) observations of Langmuir waves, which occur as broad peaks sometimes superposed with millisecond spikes. The intense Langmuir millisecond spikes

\section{TABLE I}

Summary of Observations and Predictions

\begin{tabular}{|c|c|c|c|}
\hline Date & Dec. 11,1990 & Feb 22, 1991 & Mar 7, 1991 \\
\hline Electron Density $n_{e}\left(\mathrm{~m}^{-3}\right)$ & $1.7 \times 10^{6}$ & $1.3 \times 10^{6}$ & $1.3 \times 10^{6}$ \\
\hline Ele. Plasma Freq., $f_{p e}=\omega_{p e} / 2 \pi(\mathrm{kHz})$ & 11.7 & 10.2 & 10.2 \\
\hline Electron Temperature, $T_{e}(\mathrm{~K})$ & $10^{5}$ & $7.6 \times 10^{4}$ & $4.7 \times 10^{4}$ \\
\hline Ion Temperature, $T_{i}(\mathrm{~K})$ & $5 \times 10^{4}$ & $10^{4}$ & $7 \times 10^{3}$ \\
\hline Electron Thermal Speed, $v_{T e}\left(\mathrm{~ms}^{-1}\right)$ & $1.2 \times 10^{6}$ & $1.1 \times 10^{6}$ & $8.4 \times 10^{5}$ \\
\hline Solar Wind Speed, $V_{w}\left(\mathrm{kms}^{-1}\right)$ & 382 & 310 & 401 \\
\hline Debye Length $\lambda_{D}(\mathrm{~m})$ & 17 & 17 & 13 \\
\hline Magnetic Field, B $(\gamma)$ & 6 & 2 & 2 \\
\hline Beam Speed, $v_{b}\left(\mathrm{~ms}^{-1}\right)$ & $3.5 \times 10^{7}$ & $3.5 \times 10^{7}$ & $3.5 \times 10^{7}$ \\
\hline Langmuir Wave number, $k_{L} \mathrm{~m}^{-1}$ & $2.1 \times 10^{-3}$ & $1.8 \times 10^{-3}$ & $1.8 \times 10^{-3}$ \\
\hline Relative Spectral width, $\Delta k_{L} / k_{L}$ & $0.1-0.2$ & $0.1-0.2$ & $0.1-0.2$ \\
\hline$k_{0} / k_{L}=\omega_{p e} / 65 v_{T e} k_{L}$ & 0.45 & 0.5 & 0.65 \\
\hline Peak Ele. Field of ms spikes, $E_{0}\left(\mathrm{~V}_{\mathrm{m}}^{-1}\right)$ & $9 \times 10^{-3}$ & - & $1.3 \times 10^{-2}$ \\
\hline$\frac{W_{0}}{n_{e} T_{e}}=\frac{\epsilon_{0} E_{0}^{2}}{2 n_{e} T_{e}}$ & $1.5 \times 10^{-4}$ & - & $9 \times 10^{-4}$ \\
\hline Peak Field of broad peaks, $E_{L}\left(\mathrm{Vm}^{-1}\right)$ & $3.3 \times 10^{-3}$ & $2.3 \times 10^{-3}$ & $4.6 \times 10^{-3}$ \\
\hline$\frac{W_{L}}{n_{e} T_{e}}=\frac{\epsilon_{0} E_{L}^{2}}{2 n_{e} T_{e}}$ & $2 \times 10^{-5}$ & $1.7 \times 10^{-5}$ & $1.1 \times 10^{-4}$ \\
\hline Spatial Scale of ms peaks $\Delta z(\mathrm{~m})$ & 382 & 310 & 401 \\
\hline Tra. Spa. Sca. of ms peaks $\rho \simeq v_{b} / \Omega_{e}(\mathrm{~km})$ & 33 & 99 & 99 \\
\hline EM Wave Number, $k=3.6 \times 10^{-8} f_{p e} \mathrm{~m}^{-1}$ & $4.2 \times 10^{-4}$ & $3.7 \times 10^{-4}$ & $3.7 \times 10^{-4}$ \\
\hline Har. Peak Ele. Field, $E_{t}\left(\mathrm{Vm}^{-1} \mathrm{~Hz}^{1 / 2}\right)$ & $1.6 \times 10^{-8}$ & $8 \times 10^{-9}$ & $9 \times 10^{-9}$ \\
\hline$T_{B}=2.8 \times 10^{36} E_{t}^{2} / f^{2}(\mathrm{~K})$ & $10^{12}$ & $4 \times 10^{11}$ & $5 \times 10^{11}$ \\
\hline$T_{B}(\mathrm{OTSI})(\mathrm{K})$ & $2.4 \times 10^{13}$ & $1.4 \times 10^{11}$ & $6.1 \times 10^{13}$ \\
\hline$T_{B}$ (Soliton) $(\mathrm{K})$ & $1.6 \times 10^{14}$ & $1.7 \times 10^{13}$ & $1.5 \times 10^{14}$ \\
\hline$T_{B}$ (Stochastic) (K) & $3.9 \times 10^{13}$ & $1.1 \times 10^{13}$ & $1.3 \times 10^{13}$ \\
\hline
\end{tabular}

in the solar wind were identified as collapsing solitons (Kellogg et al., 1992). The millisecond spikes associated with the present local type III events are identified as stable Langmuir solitons, whereas the broad peaks are identified as envelope solitons (Thejappa et al., 1993; 1995a, b and c). The temper- 


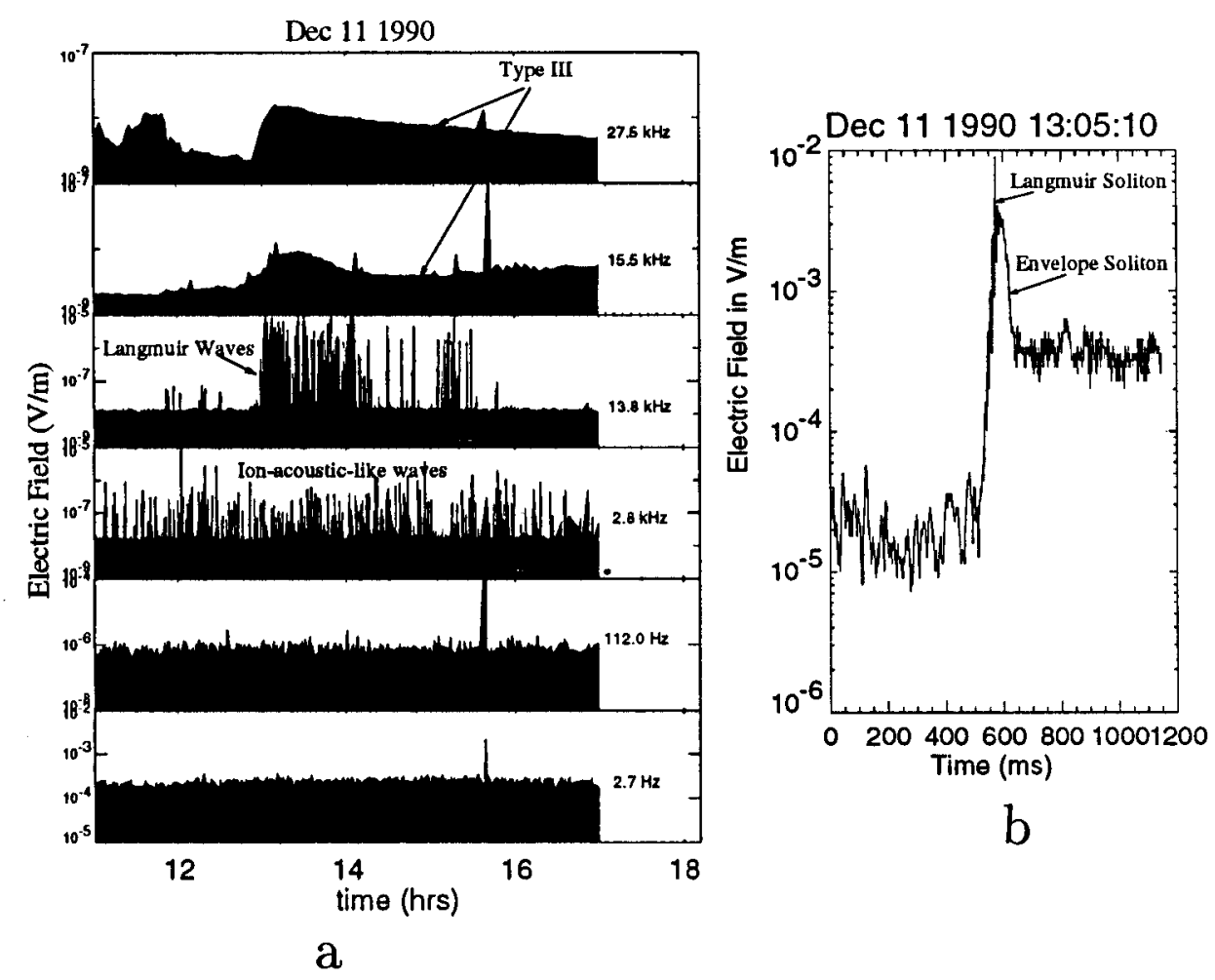

Fig. 1. Radio and in situ plasma wave phenomena

atures and the magnetic field values are taken from Ulysses archive data, made available from the NSSDC.

\section{Discussion}

The wave-wave process $L+L \pm S \rightarrow T$ is proposed as one of the efficient emission mechanisms for second harmonic radiation (Melrose et. al., 1986; Cairns and Melrose, 1985; Cairns 1987; Robinson et al., 1994), where L, S and $\mathrm{T}$ are Langmuir, ion-acoustic and electro-magnetic waves, respectively. The conditions for this process to occur are $T_{e}>5 \times 10^{5} \mathrm{~K}$ for a narrow peaked Langmuir wave distribution (Melrose, 1982) and $\frac{\Delta k_{L}}{k_{L}}>\frac{k_{0}}{k_{L}}$ (Cairns and Melrose 1985; Cairns 1987; Melrose et al 1986) for a broad Langmuir spectrum with a relative width of $\frac{\Delta k_{L}}{k_{L}}$. As seen from Table 1 , the observed $T_{e}$ values are well below $5 \times 10^{5} \mathrm{~K}$ and the $\frac{\Delta k_{L}}{k_{L}}$ values usually range from 0.1 to 0.2 (see also, Lin et. al., 1986) being much below the observed $k_{0} / k_{L}$ values, which range from 0.5 to 0.7 , indicating that $L+L \pm S \rightarrow T$ is unlikely. 
The emission process involving induced scattering is also unlikely since $k_{L}$ can become much less than $k$ in such a process (Zheleznyakov, 1977).

There are three known strong turbulence emission mechanisms, which are not restricted by any kinematic constraints: (1) oscillating-two-stream instability (Papadopoulos et al., 1974), which produces two oppositely propagating symmetric Langmuir wave distributions, (2) coherent emission by Langmuir solitons (Papadopoulos and Freund 1978) and (3) stochastic phase mixing of Langmuir waves at long wavelengths (Kruchina et. al., 1980). The emissivity $\mathrm{J}$ in these cases is $30 \pi^{3}\left(\frac{v_{T_{e}}}{c}\right)^{5}\left(\frac{W_{L}}{n_{e} T_{e}}\right)^{2} n_{e} T_{e} \omega_{p e} ; \frac{3 \sqrt{3}}{8}\left(\frac{v_{T_{e}}}{c}\right)^{4} \frac{c W_{0}}{\Delta z} \times$ $\frac{1}{k^{2} \rho^{2}}$ and $\frac{3}{32} W_{L} \omega_{p e}\left(\frac{3 T_{e}}{m_{e} c^{2}}\right)^{5 / 2}$, respectively. The brightness temperature, $T_{B}$ is related to $\mathrm{J}$ as $\kappa T_{B} \simeq \frac{(2 \pi)^{3}}{k^{2}} \frac{\mathrm{J}}{(2 \pi \Delta f)} L \simeq 9 \times 10^{44} \mathrm{~J} / f_{p e}^{2}$, where $\kappa$ is the Boltzmann constant, $L \simeq 3 \times 10^{8} \mathrm{~m}$ is the source size and $\Delta f \simeq 750 \mathrm{~Hz}$ is the bandwidth of the radio receiver. The predicted $T_{B}$ values by each of these mechanisms for the observed Langmuir wave energy densities agree very well with the actual $T_{B}$ values for a filling factor of $\simeq 0.1-0.01$ (see, Table 1 ). Since no millisecond spikes have been observed during Feb. 22 event, in some cases we have used $W_{L}$ values instead $W_{0}$ values. Thus the present observations strongly support the strong turbulence processes as the appropriate emission mechanisms at $2 \omega_{p e}$.

The research of G. T. is supported by the NASA grant NAG5-1134. We thank R. J. MacDowall for constructive comments on the manuscript.

\section{References}

Cairns, I. H.: 1987, J. Plasma Phy., 38, 179.

Cairns, I. H. and Melrose, D. B.: 1986, J. Geophys. Res., 00, 6637.

Kellogg, P. J., Goetz, K., Howard, R. L., and Monson, S. J.: 1992, Geophys. Res. Lett., $10,1303$.

Kruchina, E. N., Sagdeev, R.Z. and Shapiro, V. D.: 1980, JETP Lett., 32, 419.

Lin, R. P., Levedahl, W. K., Lotko, W., Gurnett, D. A. and Scarf, F. L.: 1986, Ap. J., 308, 954.

Melrose, D. B.: 1982, Sol. Phys., 79, 173.

Melrose, D. B., Dulk, G. A. and Cairns, I. H.: 1986, Astron. Astrophys., 163, 229.

Papadopoulos, K., Goldstein, M. L. and Smith, R. A.: 1974, Ap. J., 100, 175.

Papadopoulos, K. and Freund, H. P.:1978, Geophys. Res. Lett.,5, 881.

Stone, R. G., et al.: 1992, Astr. Ap. Suppl. Seri., 02, 291.

Reiner, M. J., Fainberg, J. and Stone, R. G.: 1992, Ap. J., 304, 340.

Robinson, P. A., Cairns, I. H. and Willes, A. J.: 1994, Ap. J, 422, 870.

Thejappa, G., Lengyel-Frey, D., Stone, R. G. and Goldstein, M. L: 1993, Ap. J.,416, 831.

Thejappa, G., Lengyel-Frey, D., Stone, R. G. and Goldstein, M. L.: 1995a, in Theoretical Geoplasma Physics, edited by T. Chang, MIT Press, Cambridge, Mass., in press.

Thejappa, G., Goldstein, M. L. Lengyel-Frey, D., Stone, R. G., MacDowall, R. J. and Papadopoulos, K .: 1995b, to be submifted to J. Geophy. Res.,

Thejappa., G., Wentzel, D., Stone, R. G.: 1995c, J. Geophys. Res.,100, 3417.

Zheleznyakov, V. V.: 1977, Electromagnetic Waves in Space Plasmas: Generation and Propagation, (Moscow: Nauka). 